\title{
Acknowledgement to Reviewers of Scientia Pharmaceutica in 2019
}

Scientia Pharmaceutica Editorial Office

MDPI, St. Alban-Anlage 66, 4052 Basel, Switzerland

Published: 16 January 2020

The editorial team greatly appreciates the reviewers who have dedicated their considerable time and expertise to the journal's rigorous editorial process over the past 12 months, regardless of whether the papers are finally published or not. In 2019, a total of 34 papers were published in the journal, with a median time to first decision of 18 days and a median time from submission to publication of 42 days. The editors would like to express their sincere gratitude to the following reviewers for their generous contribution in 2019:

$\begin{array}{ll}\text { Abdel-Tawab, Mona } & \text { Dash, Ranjeet } \\ \text { Almoazen, Hassen } & \text { Dijkstra, Bauke W. } \\ \text { Amarowicz, Ryszard } & \text { Dimitrellou, Dimitra } \\ \text { Anand, Swati } & \text { Dinu-Pirvu, Cristina-Elena } \\ \text { Antal, Diana } & \text { Domínguez Álvarez, Enrique } \\ \text { Atobe, Masakazu } & \text { Donadel, Giulia } \\ \text { Bochenek, Tomasz } & \text { Duarte, Noélia } \\ \text { Bonnet, Veronique } & \text { Durazzo, Alessandra } \\ \text { Boris, Pejin } & \text { Dziadas, Mariusz } \\ \text { Bosnea, Loulouda } & \text { Eller, Gernot } \\ \text { Botubol-Ares, Jose Manuel } & \text { Fang, Cheng } \\ \text { Bracco, Enrico } & \text { Fang, SungSoon } \\ \text { Brégier, Frédérique } & \text { Fonte, Pedro } \\ \text { Brogi, Simone } & \text { Franceschinis, Erica } \\ \text { Cardoso, Susana } & \text { Geronikaki, Athina } \\ \text { Carradori, Simone } & \text { Giner Pons, Rosa M. } \\ \text { Carullo, Gabriele } & \text { Giuffrè, Angelo Maria } \\ \text { Castñeiras, Alfonso } & \text { Gong, Hua } \\ \text { Ceranowicz, Piotr } & \text { Gonzalez-Ochoa, Guadalupe } \\ \text { Cerón-Carrasco, José Pedro } & \text { Guillemin, Jean-Claude } \\ \text { Cerra, Bruno } & \text { Hashidzume, Akihito } \\ \text { Chen, Jun } & \text { Hassan, Sherif T. S. } \\ \text { Chen, Ying } & \text { He, Hongliang } \\ \text { Cheng, Juei-Tang } & \text { Herráez-Hernández, Rosa } \\ \text { Cione, Erika } & \text { Ho, Wing Shing } \\ \text { Córdoba, Manuel } & \text { Hsieh, Chang-Wei } \\ \text { Czerwińska, Monika } & \text { Iatrou, Hermis } \\ & \end{array}$


Ionescu, Mihaela Ileana

Ionut, Ioana A.

Ita, Kevin

Iwanicki, Adam

Jara Palacios, María José

Jindrich, Jindrich

Joyasawal, Sipak

Jung, Seunho

Kalia, Yogeshvar N.

Kalwat, Michael A.

Kawakami, Susumu

Keisaku, Sato

Khairullina, Veronika

Khoddami, Ali

Kim, Il Won

Kim, Songmun

Klimov, Dmitry I.

Koike, Kenzo

Koirala, Niranjan

Kotsuchibashi, Yohei

Kowalczewski, Przemysław

Krenn, Assoc. Prof. Liselotte

Król-Kilińska, Żaneta

Kuncová, Gabriela

Kung, Chin-Ping

Länger, Reinhard

Lau-Cam, Cesar A.

Layek, Buddhadev

Lecourt, Thomas

Lee, Jong Bong

Lei, Wei

Lima, Rita de Cássia Lemos

Lins, Laurence

Liu, Yijing

Lucarini, Massimo

Manca, Maria Letizia

Manner, Suvi

Marwarha, Gurdeep

Mascini, Macello

Masłyk, Maciej

Mastinu, Andrea

Masu, Hyuma

Matsuda, Hisashi
May, Eric R.

McHugh, Kevin

Menaa, Farid

Menon, Manoj

Miroslaw, Barbara

Mohammed, Yousuf

Montenegro, Lucia

Nguyen, Quang D.

Niemirowicz-Laskowska, Katarzyna

Nitulescu, Mihai

Nociari, Marcelo

Obata, Yasuko

Oćwieja, Magdalena

Ohyama, Kaname

Olaru, Octavian Tudorel

Oliveira, Andreia

Patel, Nibedita

Petrakis, Eleftherios

Pirola, Luciano

Radominska-Pandya, Anna

Ragno, Rino

Ragusa, Andrea

Ramakumar, Kinthada

Rescifina, Antonio

Rosado, Catarina

Rudnicka, Wiesława

Salantă, Liana Claudia

Salazar, Gloria

Sanz-Paris, Alejandro

Sardu, Celestino

Saurina, Javier

Shanmugam, Muruganandan

Shikov, Alexander N.

Silva, Amelia M

Sinha, Abhijeet

Sogawa, Chiharu

Soural, Miroslav

Sreerama, Subramanya

Stringaro, Annarita

Tang, Peter H.

Tao, Kai

Telukutla, Srinivasa Reddy

Terekhova, Irina V. 
Tien, Lu-Tai

Tunesi, Marta

Tyliszczak, Bożena

Vergara, Daniele

Volkova, Elena G.

Vostinaru, Oliviu

Wagemaker, Tais Aleriana Lucon

Waldmeier, Felix

Wang, Chao-Min
Wang, Wentian

Wibowo, David

Yallapu, Murali

Yatoh, Shigeru

Yen, Feng-Lin

Yurovskaya, Marina A.

Zakłos-Szyda, Malgorzata

Zhong, Wei-Zhu

Zoltan-Istvan, Szabo

(C) 2020 by the author. Licensee MDPI, Basel, Switzerland. This article is an open access article distributed under the terms and conditions of the Creative Commons Attribution (CC BY) license (http://creativecommons.org/licenses/by/4.0/). 